\title{
Comparison of biparametric and multiparametric MRI in the diagnosis of prostate cancer
}

Lili Xu ${ }^{1 \dagger}$, Gumuyang Zhang ${ }^{1 \dagger}$, Bing Shi ${ }^{1}$, Yanhan Liu', Tingting Zou', Weigang Yan², Yu Xiao ${ }^{3}$, Huadan Xue', Feng Feng ${ }^{1}$, Jing Lei ${ }^{1}$, Zhengyu Jin ${ }^{1 *}$ and Hao Sun ${ }^{1^{*}}$ (D)

\begin{abstract}
Purpose: To compare the diagnostic accuracy of biparametric MRI (bpMRI) and multiparametric MRI (mpMRI) for prostate cancer (PCa) and clinically significant prostate cancer (csPCa) and to explore the application value of dynamic contrast-enhanced (DCE) MRI in prostate imaging.
\end{abstract}

Methods and materials: This study retrospectively enrolled 235 patients with suspected PCa in our hospital from January 2016 to December 2017, and all lesions were histopathologically confirmed. The lesions were scored according to the Prostate Imaging Reporting and Data System version 2 (PI-RADS V2). The bpMRI (T2-weighted imaging [T2WI], diffusion-weighted imaging [DWI]/apparent diffusion coefficient [ADC]) and mpMRI (T2WI, DWI/ADC and DCE) scores were recorded to plot the receiver operating characteristic (ROC) curves. The area under the curve (AUC), accuracy, sensitivity, specificity, negative predictive value (NPV), and positive predictive value (PPV) for each method were calculated and compared. The patients were further stratified according to bpMRI scores (bpMRI $\geq 3$, and $\mathrm{bpMRI}=3,4,5)$ to analyse the difference in DCE MRI between PCa and non-PCa lesions (as well as between csPCa and non-csPCa).

Results: The AUC values for the bpMRI and mpMRI protocols for PCa were comparable $(0.790$ [0.732-0.840] and 0.791 [0.733-0.841], respectively). The accuracy, sensitivity, specificity, PPV and NPV of bpMRI for PCa were 76.2, 79.5, $72.6,75.8$, and $76.6 \%$, respectively, and the values for mpMRI were $77.4,84.4,69.9,75.2$, and $80.6 \%$, respectively. The AUC values for the bpMRI and mpMRI protocols for the diagnosis of csPCa were similar (0.781 [0.722-0.832] and 0.779 [0.721-0.831], respectively). The accuracy, sensitivity, specificity, PPV and NPV of bpMRI for csPCa were 74.0, $83.8,66.9,64.8$, and $85.0 \%$, respectively; and $73.6,87.9,63.2,63.2$, and 87.8\%, respectively, for mpMRI. For patients with bpMRI scores $\geq 3$, positive DCE results were more common in PCa and csPCa lesions (both $P=0.001$ ). Further stratification analysis showed that for patients with a bpMRI score $=4, \mathrm{PCa}$ and csPCa lesions were more likely to have positive DCE results ( $P=0.003$ and $P<0.001$, respectively).

Conclusion: The diagnostic accuracy of bpMRI is comparable with that of mpMRI in the detection of PCa and the identification of csPCa. DCE MRI is helpful in further identifying PCa and csPCa lesions in patients with bpMRI $\geq 3$, especially $\mathrm{bPMRI}=4$, which may be conducive to achieving a more accurate PCa risk stratification. Rather than omitting DCE, we think further comprehensive studies are required for prostate MRI.

Keywords: Prostate cancer, Magnetic resonance imaging, Dynamic contrasted-enhanced imaging, Prostate imaging reporting and data system

\footnotetext{
*Correspondence: sunhao_robert@126.com; jinzy@pumch.cn

${ }^{\dagger}$ Lili Xu and Gumuyang Zhang contributed equally to this work.

'Department of Radiology, Peking Union Medical College Hospital, Peking

Union Medical College, Chinese Academy of Medical Sciences, Shuaifuyuan

No.1, Wangfujing Street, Dongcheng District, Beijing 100730, China

Full list of author information is available at the end of the article
}

(c) The Author(s). 2019 Open Access This article is distributed under the terms of the Creative Commons Attribution 4.0 International License (http://creativecommons.org/licenses/by/4.0/), which permits unrestricted use, distribution, and reproduction in any medium, provided you give appropriate credit to the original author(s) and the source, provide a link to the Creative Commons license, and indicate if changes were made. The Creative Commons Public Domain Dedication waiver (http://creativecommons.org/publicdomain/zero/1.0/) applies to the data made available in this article, unless otherwise stated. 


\section{Introduction}

Prostate cancer $(\mathrm{PCa})$ is the most frequently diagnosed cancer among men in over one-half of the countries of the world [1], and it is also the second leading cause of cancer-related death in the United States [2]. The ageadjusted incidence rates of $\mathrm{PCa}$ in developing Western countries and Asia has increased progressively with time $[3,4]$. The early and accurately diagnosis of $\mathrm{PCa}$ and identification of clinically significant $\mathrm{PCa}$ (csPCa), which requires more positive treatment, for reducing mortality due to aggressive $\mathrm{PCa}$ and avoid unnecessary treatment remains challenging in clinical practice.

Multiparametric magnetic resonance imaging (mpMRI) has emerged as an important tool in the early diagnosis of PCa and is particularly helpful in the detection, local staging, and estimation of the aggressiveness of prostate cancer lesions $[5,6]$. mpMRI is currently recognized as the best imaging method for assessing prostate cancer $[7,8]$. According to the recommendations of the Prostate Imaging Reporting and Data System version 2 (PI-RADS V2), mpMRI includes T1- and T2-weighted imaging (T2WI), diffusion-weighted imaging (DWI) and dynamic contrast-enhanced (DCE) MRI [9]. However, the value of DCE MRI in the detection of prostate cancer is still controversial. Some studies have shown that combining DCE MRI with T2WI and DWI does not significantly improve the diagnostic accuracy of prostate cancer [10-12]. Because DCE MRI is a time-consuming process with additional costs and a potential risk of nephrogenic systemic fibrosis, a biparametric MRI (bpMRI) protocol has been proposed. This imaging protocol omits the DCE MRI and only evaluates the T2WI and DWI sequences of the prostate $[10,13,14]$. Nevertheless, some studies have found that DCE MRI is highly sensitive in the diagnosis of PCa $[15,16]$, especially in peripheral lesions, and combining DCE MRI with DWI can significantly improve the accuracy of cancer detection [17]. Presently, in PI-RADS V2, DCE MRI can be used to upgrade lesions graded as PI-RADS 3 to PI-RADS 4 in the peripheral zone.

This study was designed to compare the diagnostic performance of bpMRI and mpMRI for PCa and csPCa, and to further explore the added value of DCE MRI in combination with T2WI and DWI in the evaluation of prostate lesions.

\section{Methods}

\section{Patients}

Patients who underwent prostate mpMRI for suspected prostate cancer at Peking Union Medical College Hospital from January 2016 to December 2017 were retrospectively enrolled in this study. Ethics approval was obtained and informed consent was waived for this study. Inclusion criteria: (1) standardized prostate
mpMRI performed for all patients; (2) standardized prostatic biopsy and/or prostatectomy performed after MRI examination and their pathological results made available; and (3) no radiation therapy, hormonal therapy or other treatments prior to MRI examination. Exclusion criteria: (1) unsatisfactory quality of MRI images; (2) biopsy or other therapies performed before MRI examination; and (3) pathological results unavailable. This study finally enrolled 235 patients; 180 underwent biopsy, and 55 underwent prostatectomy.

\section{MRI protocol}

A 3.0 T MRI scan system (GE750, GE Healthcare) with an abdominal eight-channel surface phased array coil was used to perform the imaging. The pulse sequences and MR imaging acquisition parameters applied in this study are shown in Table 1. Transverse, sagittal, and frontal T2WI images, DWI images with multiple b values and corresponding ADC maps were obtained for analysis. DCE images were obtained after intravenous injection of gadopentetate dimeglumine (Magnevist; Bayer Healthcare) at a dose of $0.1 \mathrm{mmol} / \mathrm{kg}$ of body weight and a rate of $3 \mathrm{~mL} / \mathrm{sec}$ by using an automatic injector (Spectris Solaris EP; Medrad).

\section{Image analysis}

Two radiologists experienced in interpreting prostate MRI (5 and 13 years) who were blinded to the pathological results reviewed the MRI images according to PIRADS V2 and recorded the scores from the T2WI, DWI and DCE MRI for each lesion (Fig. 1). First, the radiologists analysed T2WI and DWI images and ADC maps for each patient and recorded the T2WI score and DWI/ADC score separately. For transition zone lesions, the combined T2WI and DWI/ADC score was sufficient for categorization. For peripheral zone lesions, as suggested by the PI-RADS for cases "without adequate DCE", the categories were determined by DWI assessment alone. Subsequently, DCE images were supplied to the radiologists for assessment without a rest period. Peripheral lesions with bpMRI PI-RADS category 3 were then upgraded to PI-RADS category 4 when early enhancement was observed on DCE MRI. For controversial cases, the two radiologists jointly negotiated and reached an agreement for both the bPMRI score and mpMRI score.

\section{Standard of references}

The histological grading method for PCa was performed using the Gleason grading system [18]. As proposed in the PI-RADS V2, csPCa is defined as a tumor with a Gleason score $\geq 7$ and/or a volume $\geq 0.5 \mathrm{~cm}^{3}$ and/or extra-prostatic invasion [9]. When multiple lesions were present, the lesion with the highest PI-RADS score was used in the statistical analysis. To match the lesions on 
Table 1 Sequence parameters for prostate multiparametric MRI

\begin{tabular}{llll}
\hline Parameters & T2WI & DWI & DCE \\
\hline Sequence & FRFSE & SE-EPI & 3 D-GRE \\
TR/TE (ms) & $4137 / 86$ & $4200 / 90$ & $4.3 / 1.3$ \\
Flip angle (degree) & 110 & 90 & 12 \\
Echo train length & 32 & 1 & $\mathrm{~N} / \mathrm{A}$ \\
Field of view (mm $\times$ & $270 \times$ & $360 \times 360$ & $400 \times 400$ \\
mm) & 270 & & $320 \times 192$ \\
Matrix size & $288 \times$ & $128 \times 96$ & 3.0 \\
& 192 & & \\
Thickness (mm) & 3.0 & 3.0 & Temporal resolution < $<10$ s, total scan time of 5 \\
Other & & b values $=100,150,200,500,800,1000,1500,2000 \mathrm{~mm}^{2} /$ & min \\
\hline
\end{tabular}

TR Repetition time, TE Time echo, FRFSE Fast relaxation fast spin echo, SE-EPI Spin-echo echo planar imaging, 3D-GRE 3D-gradient echo

MR images with histopathologic specimens from biopsy or prostatectomy, we first roughly located the highest PI-RADS category lesions on MR images to the corresponding 11-region map and then obtained the biopsy/prostatectomy results for the relevant regions.

\section{Statistical analysis}

Using the pathological results as the standard of reference, the receiver operating characteristic (ROC) curves for bpMRI and mpMRI were plotted, and the diagnostic accuracy, sensitivity, specificity, positive predictive value (PPV) and negative predictive value (NPV) and their 95\% confidence intervals $(\mathrm{CI})$ were calculated to evaluate the diagnostic performance of the two scoring methods. A Delong test was used to assess the difference between areas under the curve (AUC). In detail, for the accuracy in detecting $\mathrm{PCa}$ or csPCa, PI-RADS V2 category 1-3 were considered negative, while PI-RADS V2 category 4-5 were considered positive. Patients with bpMRI PIRADS $\geq 3$ were further stratified according to bpMRI category to assess the difference in DCE MRI between $\mathrm{PCa}$ and non-PCa lesions (as well as between csPCa and non-csPCa). The chi-square test or Fisher's exact test was used to test for differences in dichotomized score values. The difference was considered statistically significant with a two-sided $P$ value $<0.05$. Statistical analysis was performed using SPSS 22.0 (IBM) and MedCal 15.0 software (MedCalc Software).

\section{Results}

\section{Clinicopathological data}

Figure 2 shows a flowchart of patient recruitment. With a mean age of $66.87 \pm 8.53$ years and a median PSA level of $4.65(0.22-86.00) \mathrm{ng} / \mathrm{ml}$, a total of 235 patients who met the criteria mentioned above were enrolled in this study. Histopathological analysis revealed PCa in 122 (51.9\%) of these patients and non-PCa in 113 (48.1\%). Among the 122 patients with $\mathrm{PCa}, 99$ were defined as csPCa $(67$ with Gleason score $=3+4$, and 32 with Gleason score $\geq 4+4$ ) (Table 2).
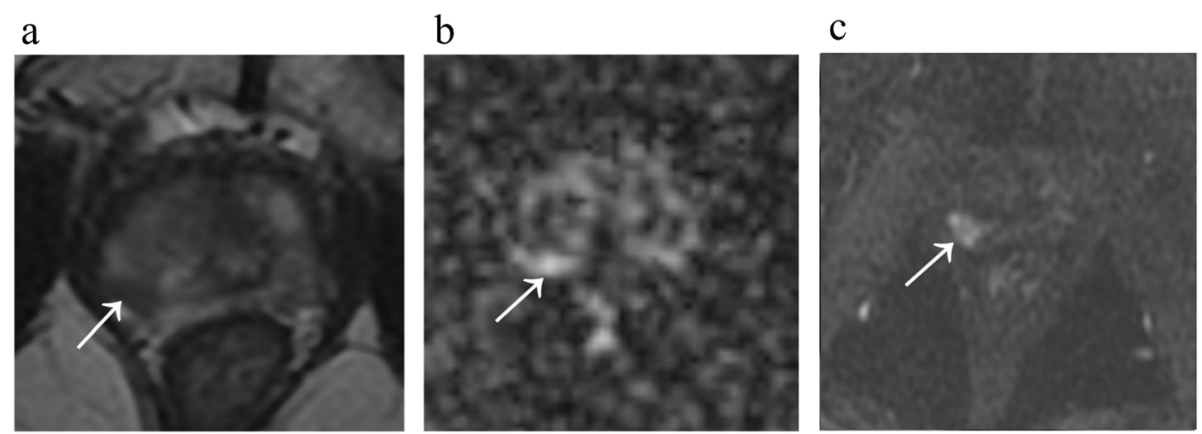

Fig. 1 Images from a 73-year-old man with a PSA level of $8.4 \mathrm{ng} / \mathrm{mL}$. a A focal hypointensity with a partially circumscribed margin is shown in the right posterior peripheral zone on axial T2-weighted MRI, with a T2WI score of 4. b DWI shows slightly increased signal intensity of the lesion with a score of 3. c DCE MRI reveals the lesion with an early and clear enhancement, which translates to a positive DCE score. The PI-RADS category of this lesion is 3 with the bpMRI protocol and 4 with the mpMRI protocol. The lesion was proven to be a clinically significant cancer with a Gleason Score $=4+3$ by biopsy 


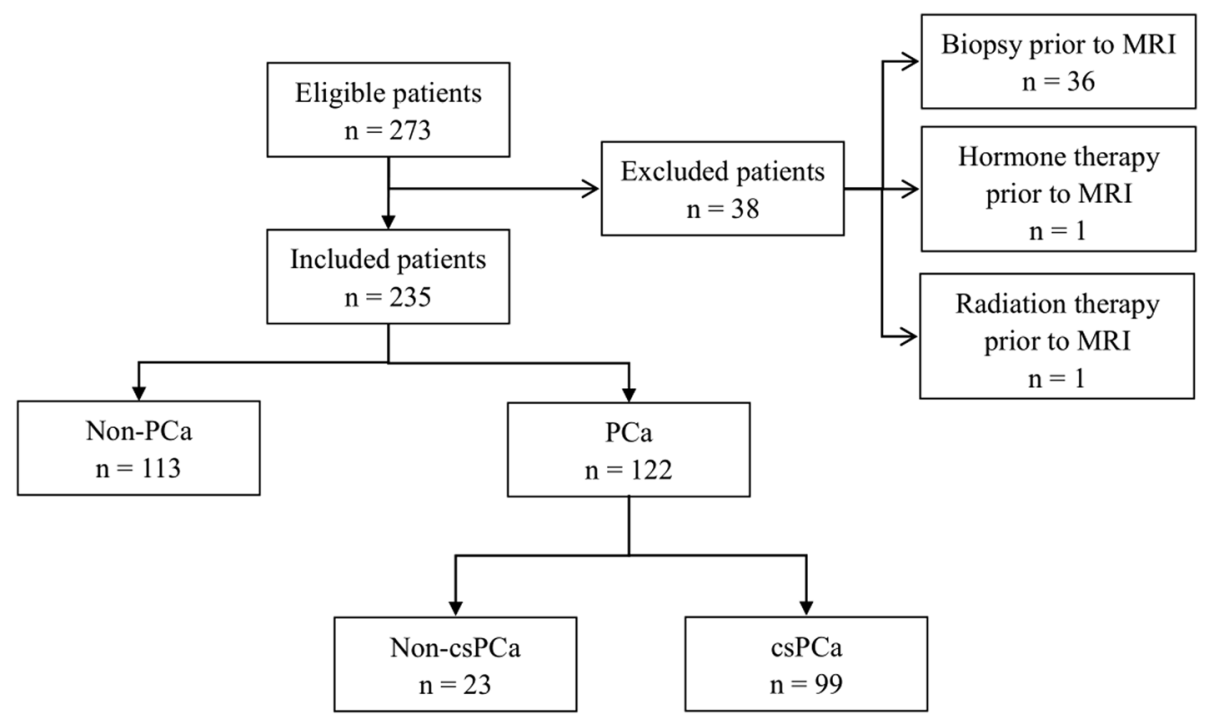

Fig. 2 Flow diagram of the inclusion and exclusion criteria in this research

Comparison of the diagnostic performances of bpMRI and mpMRI

The AUCs for the bpMRI and mpMRI protocols in diagnosing PCa were 0.790 (0.732-0.840) and 0.791 (0.7330.841 ), respectively, and the difference was not statistically significant $(P>0.05)$. The accuracy, sensitivity, specificity, PPV and NPV of bpMRI for diagnosing PCa were $76.2,79.5,72.6,75.8$, and $76.6 \%$, respectively; the values for mpMRI were $77.4,84.4,69.9,75.2$, and $80.6 \%$, respectively. For the diagnosis of csPCa, the AUC values of bPMRI and mpMRI were $0.781(0.722-0.832)$ and 0.779 (0.721-0.831), respectively, which were not significantly different $(P>0.05)$. The accuracy, sensitivity,

Table 2 Clinicopathological data of patients included in this study

\begin{tabular}{ll}
\hline Clinicopathological data & All patients $(n=235)$ \\
\hline Age (year), mean \pm SD & $66.87 \pm 8.53$ \\
PSA (ng/mL), median (range) & $4.65(0.22-86.00)$ \\
PCa, $n(\%)$ & $122(51.9)$ \\
csPCa, $n(\%)$ & $99(42.1)$ \\
Gleason score, $n(\%)$ & \\
$3+3$ & $23(9.8)$ \\
$3+4$ & $42(17.8)$ \\
$4+3$ & $25(10.6)$ \\
$3+5$ & $1(0.4)$ \\
$4+4$ & $9(3.8)$ \\
$4+5$ & $15(6.4)$ \\
$5+4$ & $5(2.1)$ \\
$5+5$ & $2(0.9)$ \\
\hline
\end{tabular}

specificity, PPV and NPV of bpMRI were 74.0, 83.8, $66.9,64.8$, and $85.0 \%$, respectively, and the values for mpMRI were $73.6,87.9,63.2,63.2$, and $87.8 \%$, respectively (Table 3 and Fig. 3).

\section{DCE score for the diagnosis of PCa and csPCa}

For patients with bpMRI scores $\geq 3$, the DCE score was statistically significant between $\mathrm{PCa}$ and non-PCa and between csPCa and non-csPCa patients (both $P=0.001$ ). That is, DCE-positive results were more common in $\mathrm{PCa}$ and $\mathrm{csPCa}$ lesions among these patients. After stratifying according to bpMRI score, the difference in DCE between PCa and non-PCa and between csPCa and non-csPCa was statistically significant in patients with bpMRI score $=4(P=0.003$, and $P<0.001$, respectively $)$, which means that among these patients, $\mathrm{PCa}$ and $\mathrm{csPCa}$ lesions were more likely to have early enhancement in DCE MRI. However, for patients with $b p M R I=3$ and $\mathrm{bpMRI}=5$, no significant difference was noted (all $P>$ 0.05) (Table 4).

\section{Discussion}

The results of this study indicate that the bpMRI protocol is comparable to the mpMRI protocol in the diagnosis of PCa and csPCa. For patients with a bpMRI score $\geq$ 3 , the DCE score was significantly different between PCa and non-PCa and between csPCa and non-csPCa. Further stratification analysis showed that in patients with a bpMRI score $=4$, a significant difference was also noted in the DCE score. The results indicated that $\mathrm{PCa}$ and csPCa lesions were more likely to have positive DCE results in bpMRI $\geq 3$ patients (especially bpMRI $=4$ patients), suggesting that for these patients, the application of DCE MRI may help to improve the tumor detection 
Table 3 Comparison of mpMRI and bpMRI for diagnosing prostate lesions

\begin{tabular}{|c|c|c|c|c|c|}
\hline & mpMRI & & bpMRI & & $P$ \\
\hline & $<4(n=98)$ & $\geq 4(n=137)$ & $<4(n=107)$ & $\geq 4(n=128)$ & \\
\hline Non-PCa, n (\%) & $79(80.6)$ & $34(24.8)$ & $82(76.6)$ & $31(24.2)$ & \\
\hline PCa, n (\%) & 19 (19.4) & $103(75.2)$ & $25(23.4)$ & $97(75.8)$ & \\
\hline Accuracy & & $77.4(71.7-82.3)$ & & $76.2(70.3-81.2)$ & \\
\hline Sensitivity & & $84.4(76.8-90.4)$ & & 79.5 (71.3-86.3) & \\
\hline Specificity & & $69.9(60.6-78.2)$ & & $72.6(63.4-80.5)$ & \\
\hline PPV & & $75.2(67.1-82.2)$ & & $75.8(67.4-82.9)$ & \\
\hline NPV & & $80.6(71.3-87.9)$ & & $76.6(67.5-84.3)$ & \\
\hline AUC & & $0.791(0.733-0.841)$ & & $0.790(0.732-0.840)$ & $0.760^{*}$ \\
\hline Non-csPCa, n (\%) & $86(87.8)$ & $50(36.5)$ & $91(85.0)$ & $45(35.2)$ & \\
\hline csPCa, n (\%) & $12(12.2)$ & $87(63.5)$ & $16(15.0)$ & $83(64.8)$ & \\
\hline Accuracy & & $73.6(67.6-78.9)$ & & $74.0(68.1-79.2)$ & \\
\hline Sensitivity & & 87.9 (79.8-93.6) & & $83.8(75.1-90.5)$ & \\
\hline Specificity & & $63.2(54.5-71.3)$ & & $66.9(58.3-74.7)$ & \\
\hline PPV & & $63.2(54.9-71.6)$ & & $64.8(55.9-73.1)$ & \\
\hline NPV & & $87.8(79.6-93.5)$ & & $85.0(76.8-91.2)$ & \\
\hline AUC & & $0.779(0.721-0.831)$ & & $0.781(0.722-0.832)$ & $0.753^{*}$ \\
\hline
\end{tabular}

The accuracy, sensitivity, specificity, positive predictive value (PPV) and negative predictive value (NPV) are presented as (\%, [95\% confidence interval]); the areas under the curve (AUCs) are presented with 95\% confidence intervals

*Delong test was used to compare the AUCs of bpMRI and mpMRI

rate and achieve a more accurate tumor aggressiveness classification.

Currently, mpMRI is the standard method for prostate imaging and plays an important role in the detection, targeted biopsy, local staging and risk classification of prostate cancers. PI-RADS has been proposed to standardize the scan process, image interpretation and report writing of prostate mpMRI, to improve the management of prostate cancer patients [9]. Compared with the PI-RADS proposed in 2012, the main change in PI-
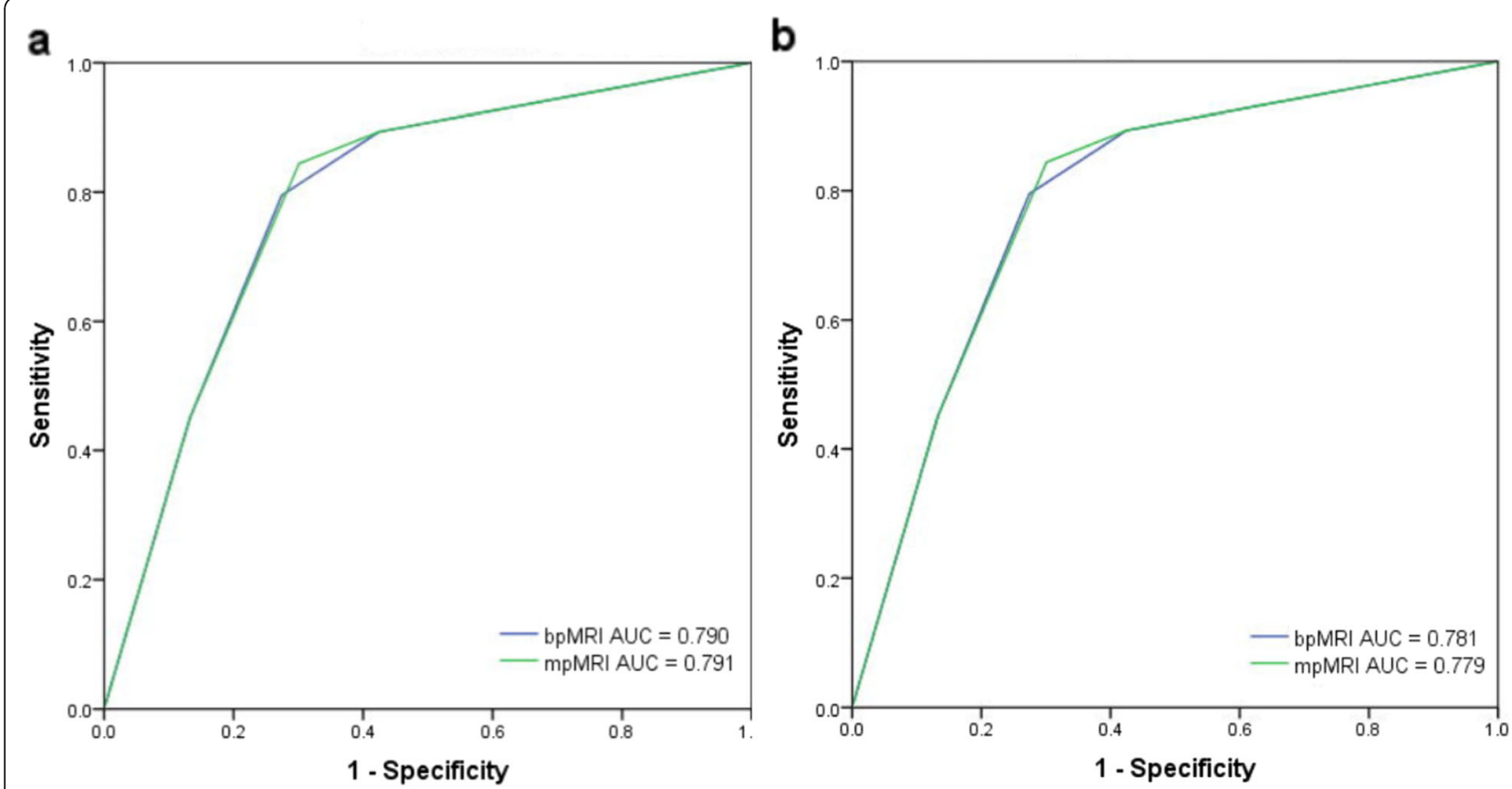

Fig. 3 Comparison of ROC curves between bPMRI and mpMRI for prostate cancer (a) and clinically significant cancer detection (b) 
Table 4 The differences in DCE in patients with bpMRI $\geq 3(n, \%)$

\begin{tabular}{|c|c|c|c|c|c|c|}
\hline & Non-PCa $(n=48)$ & PCa $(n=109)$ & $P^{*}$ & Non-csPCa $(n=66)$ & $\operatorname{csPCa}(n=91)$ & $P^{*}$ \\
\hline bpMRI $\geq 3(n=157)$ & & & 0.001 & & & 0.001 \\
\hline DCE $(-)$ & 19 (39.6) & 17 (15.6) & & $24(36.4)$ & $12(13.2)$ & \\
\hline DCE $(+)$ & $29(60.4)$ & $92(84.4)$ & & $42(63.6)$ & 79 (86.8) & \\
\hline bpMRI = $3(n=29)$ & & & 0.286 & & & 0.943 \\
\hline DCE $(-)$ & $9(52.9)$ & $4(33.3)$ & & $10(47.6)$ & $3(37.5)$ & \\
\hline DCE $(+)$ & $8(47.1)$ & $8(66.7)$ & & $11(52.4)$ & $5(62.5)$ & \\
\hline bpMRI $=4(n=58)$ & & & 0.003 & & & $<0.001$ \\
\hline DCE $(-)$ & $9(56.3)$ & $6(14.3)$ & & $12(52.2)$ & $3(8.6)$ & \\
\hline DCE $(+)$ & $7(43.8)$ & $36(85.7)$ & & $11(47.8)$ & $32(91.4)$ & \\
\hline $\mathrm{bpMRI}=5(n=70)$ & & & 0.844 & & & 0.991 \\
\hline DCE $(-)$ & $1(6.7)$ & $7(12.7)$ & & $2(9.1)$ & $6(12.5)$ & \\
\hline DCE $(+)$ & $14(93.3)$ & $48(87.3)$ & & $20(90.9)$ & $42(87.5)$ & \\
\hline
\end{tabular}

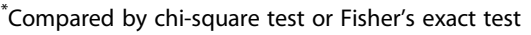

RADS V2 is the proposal of a dominant sequence, in which T2WI is proposed as the dominant sequence for transition zone lesions and DWI for peripheral zone lesions. DCE MRI plays a supplementary role only when DWI is not sufficient for diagnosis [19, 20], and DCE positivity only upgrades DWI category 3 lesions in the peripheral zone to category 4 .

Considering the long acquisition time of DCE MRI, its potential risks and additional costs, patient discomfort associated with contrast agent injection, and the relatively small incremental benefit of DCE MRI, several authors have suggested that it be abandoned [21, 22]. Some studies have compared the diagnostic performance of bpMRI and mpMRI [13, 23]. The research of Junker et al. [23] showed that bpMRI and mpMRI have comparable diagnostic performance in detecting prostate cancer (AUC $=0.914$ and 0.917 , respectively). Their comparability was also demonstrated by some metaanalyses published recently [24-28]. From a head-tohead comparison in detecting PCa, mpMRI had a significantly higher pooled sensitivity than bpMRI [25]. Kuhl et al. [13] investigated 542 patients with PSA $\geq 3 \mathrm{ng} / \mathrm{mL}$ and negative transrectal ultrasonography-guided biopsy findings, and found that bPMRI and mpMRI have similar accuracy in diagnosing clinically significant cancer $(89.1 \%$ vs. $87.2 \%)$; between-reader agreement for the bpMR protocol was substantial $(k=0.81)$, while the agreement for mpMRI was moderate $(\mathrm{k}=0.60)$. Our results also indicated the comparability of bpMRI and mpMRI in the detection of prostate cancer and the identification of clinically significant lesions.

Whether DCE imaging has added benefits in the diagnosis and risk stratification of prostate cancer has also been widely studied. Greer et al. [17] reported that in the peripheral zone, DCE positivity improved the probability of cancer detection of PI-RADS category 2, 3, and
4 lesions (OR, 2.00; $P=0.027$ ); for these lesions, the tumor detection rate increased by $15.7,16.0$, and $9.2 \%$, respectively. These results suggest that the DCE sequence has important application value in the diagnosis of prostate cancer in the peripheral zone, which may help improve the risk stratification accuracy of prostate cancer. However, since the number of transition zone lesions was limited in this study, DCE was not found to significantly increase the tumor detection rate in the transition zone. The study by Rosenkrantz et al. [29] analysed the role of the DCE sequence in the transition zone. This study selected 106 patients with suspected $\mathrm{PCa}$, and three radiologists scored the transition zone using the pathological results from prostatectomy as a reference. The results showed that scoring according to T2WI + DWI had higher sensitivity than depending on T2WI alone, but the addition of DCE did not improve the diagnostic sensitivity, so DCE was considered useless in the analysis of transition zone lesions. Our study showed that, for patients with bpMRI category $\geq 3$, DCE was noted to be statistically significant in the diagnosis of prostate cancer and the identification of clinically significant disease. Further stratification analysis showed a significant difference in DCE between $\mathrm{PCa}$ and non-PCa and between csPCa and non-csPCa among patients with bPMRI category $=4$, which indicates that for those patients, DCE may help improve the tumor detection rate and the accuracy of tumor aggressiveness classification. The research of Rosenkrantz et al. [30] proposed some adjustments to the decision rules of PI-RADS and found that for transition zone lesions upgraded from category 3 to 4 based on a DCE score of positive when integrating new criteria (unencapsulated sheet-like enhancement), the frequency of Gleason score $\geq 7$ tumors was $33.3-57.1 \%$. Rather than omitting DCE, further comprehensive studies are required for future updates of the existing system. 
There are some limitations in this study. First, the sample size of our study is not very large. Second, due to the limited sample size, we did not separate lesions into peripheral and transition zones and investigate the added value of DCE in different zones. Third, most patients had theire biopsy results used as the standard of reference, which may have underestimated the diagnostic performance of mpMRI and bpMRI due to the high false negative rate of biopsy [31]. Further studies including more patients are ongoing to verify the conclusion of this study and analyse the added value of DCE MRI in different zones. In addition to providing qualitative parameters, DCE MRI can also provide a wealth of quantitative and semi-quantitative parameters [32]. Whether these other parameters are useful in the early diagnosis of prostate cancer also needs to be tested.

\section{Conclusion}

In conclusion, the results of this study indicated that bPMRI is comparable to mpMRI in the diagnosis of $\mathrm{PCa}$ and csPCa, but DCE MRI is helpful in further identifying $\mathrm{PCa}$ and csPCa in patients with bpMRI $\geq 3$, especially $\operatorname{bpMRI}=4$, which may help achieve a more accurate aggressiveness classification and individualized treatment of prostate cancer.

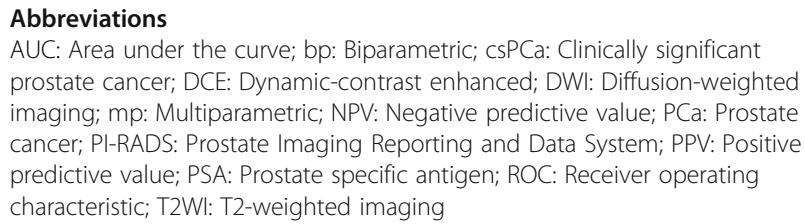
prostate cancer; DCE: Dynamic-contrast enhanced; DWI: Diffusion-weighted imaging; mp: Multiparametric; NPV: Negative predictive value; PCa: Prostate cancer; PI-RADS: Prostate Imaging Reporting and Data System; PPV: Positive predictive value; PSA: Prostate specific antigen; ROC: Receiver operating characteristic; T2Wl: T2-weighted imaging

\section{Acknowledgements}

Not applicable.

\section{Authors' contributions}

Guarantor of integrity of the entire study: HS, ZJ; study concepts and design: LX, GZ, HS; literature research: LX, BS, YL; data collection: GZ, WY, YX; data analysis: LX, TZ; manuscript preparation: LX; manuscript review: HS, HX, FF. All authors read and approved the final manuscript.

\section{Funding}

This research is supported by Beijing Municipal Natural Science Foundation under Grant No. 7192176; Fundamental Research Funds for the Central Universities under Grant No.3332018022; Education Reform Fund of Peking Union Medical College under Grant No. 10023201700104; National Natural Science Foundation of China under Grant No. 91859119; National Public Welfare Basic Scientific Research Program of Chinese Academy of Medical Sciences under Grant No. 2019PT320008 and 2018PT32003; Youth Educator Program of Peking Union Medical College under Grant No.2019zlgc0705.

\section{Availability of data and materials}

The datasets used and/or analysed during the current study are available from the corresponding author on reasonable request.

Ethics approval and consent to participate

Ethical approval was obtained by the local institutional review board. Informed consent was waived.

\section{Consent for publication}

Not applicable.

\section{Competing interests}

The authors declare that they have no competing interests.

\section{Author details}

'Department of Radiology, Peking Union Medical College Hospital, Peking Union Medical College, Chinese Academy of Medical Sciences, Shuaifuyuan No.1, Wangfujing Street, Dongcheng District, Beijing 100730, China. ${ }^{2}$ Department of Urology, Peking Union Medical College Hospital, Peking Union Medical College, Chinese Academy of Medical Sciences, Beijing 100730, China. ${ }^{3}$ Department of Pathology, Peking Union Medical College Hospital, Peking Union Medical College, Chinese Academy of Medical Sciences, Beijing 100730, China.

Received: 30 April 2019 Accepted: 6 December 2019

Published online: 21 December 2019

\section{References}

1. Bray F, Ferlay J, Soerjomataram I, Siegel RL, Torre LA, Jemal A. Global cancer statistics 2018: GLOBOCAN estimates of incidence and mortality worldwide for 36 cancers in 185 countries. CA Cancer J Clin. 2018;68(6):394-424.

2. Siegel RL, Miller KD, Jemal A. Cancer statistics, 2018. CA Cancer J Clin. 2018; 68(1):7-30.

3. Chen W, Zheng R, Baade PD, et al. Cancer statistics in China, 2015. CA Cancer J Clin. 2016:66(2):115-32.

4. Teoh JYC, Hirai HW, Ho JMW, Chan FCH, Tsoi KKF, Ng CF. Global incidence of prostate cancer in developing and developed countries with changing age structures. PLoS One. 2019;14(10):e0221775.

5. Turkbey B, Brown AM, Sankineni S, Wood BJ, Pinto PA, Choyke PL. Multiparametric prostate magnetic resonance imaging in the evaluation of prostate cancer. CA Cancer J Clin. 2016;66(4):326-36.

6. Ueno Y, Tamada T, Bist V, et al. Multiparametric magnetic resonance imaging: Current role in prostate cancer management. Int J Urol. 2016;23(7): 550-7.

7. Mottet N, Bellmunt J, Bolla M, et al. EAU-ESTRO-SIOG guidelines on prostate cancer. Part 1: screening, diagnosis, and local treatment with curative intent. Eur Urol. 2017;71(4):618-29.

8. Lam TBL, MacLennan S, Willemse PM, et al. EAU-EANM-ESTRO-ESUR-SIOG prostate cancer guideline panel consensus statements for deferred treatment with curative intent for localised prostate cancer from an international collaborative study (Detective study). Eur Urol. 2019;76(6):790-813.

9. Weinreb JC, Barentsz JO, Choyke PL, et al. PI-RADS prostate imaging reporting and data system: 2015, version 2. Eur Urol. 2016;69(1):16-40.

10. Stanzione A, Imbriaco M, Cocozza S, et al. Biparametric 3T magnetic resonance imaging for prostatic cancer detection in a biopsy-naive patient population: a further improvement of PI-RADS v2? Eur J Radiol. 2016;85(12): 2269-74.

11. Hansford BG, Peng $Y$, Jiang $Y$, et al. Dynamic contrast-enhanced MR imaging curve-type analysis: is it helpful in the differentiation of prostate cancer from healthy peripheral zone? Radiology. 2015;275(2):448-57.

12. Scialpi M, Prosperi E, D'Andrea A, et al. Biparametric versus multiparametric MRI with non-endorectal coil at 3T in the detection and localization of prostate cancer. Anticancer Res. 2017;37(3):1263-71.

13. Kuhl CK, Bruhn R, Kramer N, Nebelung S, Heidenreich A, Schrading S. Abbreviated Biparametric prostate MR imaging in men with elevated prostate-specific antigen. Radiology. 2017;285(2):493-505.

14. Di Campli E, Delli Pizzi A, Seccia B, et al. Diagnostic accuracy of biparametric vs multiparametric MRI in clinically significant prostate cancer: comparison between readers with different experience. Eur J Radiol. 2018:10117-23.

15. Girouin N, Mege-Lechevallier F, Tonina Senes A, et al. Prostate dynamic contrast-enhanced MRI with simple visual diagnostic criteria: is it reasonable? Eur Radiol. 2007;17(6):1498-509.

16. Kim CK, Park BK, Lee HM, Kwon GY. Value of diffusion-weighted imaging for the prediction of prostate cancer location at $3 T$ using a phased-array coil: preliminary results. Investig Radiol. 2007;42(12):842-7.

17. Greer MD, Shih JH, Lay N, et al. Validation of the dominant sequence paradigm and role of dynamic contrast-enhanced imaging in PI-RADS version 2. Radiology. 2017;285(3):859-69.

18. Epstein Jl, Amin MB, Reuter VE, Humphrey PA. Contemporary Gleason grading of prostatic carcinoma: an update with discussion on practical issues to implement the 2014 International Society of Urological Pathology 
(ISUP) consensus conference on Gleason grading of prostatic carcinoma. Am J Surg Pathol. 2017;41(4):e1-7.

19. Junker D, Quentin M, Nagele U, et al. Evaluation of the PI-RADS scoring system for mpMRI of the prostate: a whole-mount step-section analysis. World J Urol. 2015;33(7):1023-30.

20. Iwazawa J, Mitani T, Sassa S, Ohue S. Prostate cancer detection with MRI: is dynamic contrast-enhanced imaging necessary in addition to diffusionweighted imaging? Diagn Interv Radiol. 2011;17(3):243-8.

21. Rud E, Baco E. Jeffrey C. Weinreb, Jelle O. Barentsz, Peter L. Choyke, et al. PIRADS prostate imaging - reporting and data system: 2015, version 2. Eur Urol 2016;69:16-40. Is contrast-enhanced magnetic resonance imaging really necessary when searching for prostate cancer? Reply. Eur Urol. 2016; 70(5):E136.

22. Barentsz JO, Choyke PL, Cornud F, et al. Reply to Erik Rud and Eduard Baco's Letter to the Editor re: Re: Jeffrey C. Weinreb, Jelle O. Barentsz, Peter L. Choyke, et al. PI-RADS prostate imaging - reporting and data system: 2015, version 2. Eur Urol. 2016;69:16-40 Eur Urol. 2016;70(5):E137-E8.

23. Junker D, Steinkohl F, Fritz V, et al. Comparison of multiparametric and biparametric MRI of the prostate: are gadolinium-based contrast agents needed for routine examinations? World J Urol. 2019;37(4):691-9.

24. Alabousi M, Salameh JP, Gusenbauer K, et al. Biparametric vs multiparametric prostate magnetic resonance imaging for the detection of prostate cancer in treatment-naive patients: a diagnostic test accuracy systematic review and meta-analysis. BJU Int. 2019;124(2):209-20.

25. Niu XK, Chen XH, Chen ZF, Chen L, Li J, Peng T. Diagnostic performance of Biparametric MRI for detection of prostate cancer: a systematic review and meta-analysis. AJR Am J Roentgenol. 2018;211(2):369-78.

26. Woo S, Suh CH, Kim SY, Cho JY, Kim SH, Moon MH. Head-to-head comparison between biparametric and multiparametric MRI for the diagnosis of prostate cancer: a systematic review and meta-analysis. AJR Am J Roentgenol. 2018;211(5):W226-W41.

27. Kang Z, Min X, Weinreb J, Li Q, Feng Z, Wang L. Abbreviated biparametric versus standard multiparametric MRI for diagnosis of prostate cancer: a systematic review and meta-analysis. AJR Am J Roentgenol. 2019;212(2): $357-65$

28. Monni F, Fontanella P, Grasso A, et al. Magnetic resonance imaging in prostate cancer detection and management: a systematic review. Minerva Urol Nefrol. 2017;69(6):567-78.

29. Rosenkrantz AB, Kim S, Campbell N, Gaing B, Deng FM, Taneja SS. Transition zone prostate cancer: revisiting the role of multiparametric MRI at $3 \mathrm{~T}$. AJR Am J Roentgenol. 2015;204(3):W266-72.

30. Rosenkrantz AB, Babb JS, Taneja SS, Ream JM. Proposed adjustments to PIRADS version 2 decision rules: impact on prostate cancer detection. Radiology. 2017;283(1):119-29.

31. Drost FH, Osses D, Nieboer D, et al. Prostate magnetic resonance imaging with or without magnetic resonance imaging-targeted biopsy, and systematic biopsy for detecting prostate cancer: a cochrane systematic review and meta-analysis. Eur Urol. 2020;77(1):78-94.

32. Sanz-Requena R, Marti-Bonmati L, Perez-Martinez R, Garcia-Marti G. Dynamic contrast-enhanced case-control analysis in 3T MRI of prostate cancer can help to characterize tumor aggressiveness. Eur J Radiol. 2016;85(11):2119-26.

\section{Publisher's Note}

Springer Nature remains neutral with regard to jurisdictional claims in published maps and institutional affiliations.

Ready to submit your research? Choose BMC and benefit from:

- fast, convenient online submission

- thorough peer review by experienced researchers in your field

- rapid publication on acceptance

- support for research data, including large and complex data types

- gold Open Access which fosters wider collaboration and increased citations

- maximum visibility for your research: over $100 \mathrm{M}$ website views per year

At $\mathrm{BMC}$, research is always in progress.

Learn more biomedcentral.com/submissions 